\title{
MicroRNA-32-5p Inhibits Metastasis By Directly Targeting VPS4B and Increases Sensitivity to Dihydroartemisinin in Neuroblastoma
}

\section{Lina Chen}

China Academy of Chinese Medical Sciences

Hui Liu

China Academy of Chinese Medical Sciences

\section{Zhongyuan Zheng}

China Academy of Chinese Medical Sciences

\section{Xi Wang}

China Academy of Chinese Medical Sciences

\section{Shuiqing Qu}

China Academy of Chinese Medical Sciences

\section{Yuanmin Yang}

China Academy of Chinese Medical Sciences

\section{Shuoqiu Deng}

China Academy of Chinese Medical Sciences

\section{Yu Zhang}

China Academy of Chinese Medical Sciences

\section{Tuo Liu}

China Academy of Chinese Medical Sciences

\section{Honghua Cui}

School of Traditional Chinese Medicine, Guangdong Pharmaceutical University

\section{Yujie Li ( $\nabla$ yjli@icmm.ac.cn )}

China Academy of Chinese Medical Sciences

\section{Research Article}

Keywords: neuroblastoma metastasis, miR-32-5p, dihydroartemisinin, VPS4B

Posted Date: January 26th, 2022

DOI: https://doi.org/10.21203/rs.3.rs-1233808/v1 
License: (c) (i) This work is licensed under a Creative Commons Attribution 4.0 International License. Read Full License 


\section{Abstract}

Neuroblastoma (NB) is a malignant pediatric tumor requiring new treatments. Accumulating evidence has confirmed that microRNAs (miRNAs) have a major effect on NB metastasis. The present study aimed to characterize a novel miRNA, miR-32-5p, by exploring its expression, function, as well as action mechanism in NB metastasis. Real-time quantitative polymerase chain reaction (qRT-PCR) as well as Western blotting was utilized to measure the expression of miR-32-5p and its target-vacuolar protein sorting 4B (VPS4B). Transwell assay was conducted to test the cell migration as well as its invasion in vitro, and the metastasis xenograft model of nude mice was set up by injections through the caudal vein in vivo. In comparison with the normal tissue and cell line, the miR-32-5p expression showed a marked downregulation in human NB samples and cell lines. Furthermore, the miR-32-5p upregulation suppressed NB cell metastasis in culture and the tumor xenograft model. Although in NB cell lines, VPS4B was found to be miR-32-5p's direct target, its overexpression would reverse miR-32-5p's suppressive effects. Moreover, miR-32-5p increased the sensitivity to dihydroartemisinin in neuroblastoma. The current outcomes implicated that the axis of miR-32-5p/VPS4B can be NB metastasis's novel therapeutic target.

\section{Introduction}

Neuroblastoma (NB), originating from neuroectodermal, is a typical extracranial tumor in childhood [1]. As the most common deadly malignancy in infancy, it occupies about $15 \%$ of mortality in pediatric cancer [2]. The NB clinical manifestations are highly heterogeneous, ranging from a mass in a different place to an aggressive high-risk disease due to substantial tumor metastasis [3]. Numerous therapy methods have been developed, including medical surgery, radiation therapy, and immunotherapy, as well as chemotherapeutics and autologous stem cell transplantation, The selection of methods depends on the patient's age, tumor biology, and stage at diagnosis [4]. In recent years, we have made powerful progress in clinical treatment, and survival rates for early-stage patients have been improved slightly, but the therapies for high-risk groups and metastatic NB are poor [5]. Therefore, improvement in the patient survival rate depends on the recognition of new targets and the development of novel treatment approaches.

As the main active metabolite of artemisinin derivatives [6], dihydroartemisinin (DHA) has significant antitumor activities on various human cancers. Recently, a study founded that DHA suppresses the multiplication of K562 leukemia cells by knocking down the PKM2 and GLUT1 expression to inhibit aerobic glycolysis [7]. Moreover, DHA can contribute to the cell cycle arrest and curb the carcinoma cell viability in the head and neck by inducing ferroptosis [8]. Nevertheless, the mechanisms by which DHA inhibits tumor progression and metastasis have not been fully elucidated [9]. The putative mechanisms include inhibiting angiogenesis [10], inducing autophagy [11], inhibiting proliferation [12], inducing apoptosis [13], involving oxidative stress response [14], and inhibiting invasion and metastasis [15]. Therefore, DHA is speculated to inhibit advanced metastasis of NB, and the related mechanisms are yet unclear. 
MicroRNAs (miRNAs), containing 18-20 nucleotides, are small non-coding RNA molecules found in eukaryotes [16]. They are essential in the regulation of posttranscriptional genes [17]. Several studies have found that miRNAs control various biological processes by regulating the oncogene expression [18], participating in cell cycle control, differentiation, metabolism, apoptosis, migration, invasion, tumorigenesis, and angiogenesis [19]. miR-32-5p is reported to promote inflammatory challenges within the brain and dysfunctional of nerve fibers via regulating dual-specificity phosphatase 5 [20]. In addition, by downregulating the PTEN expression, miR-32-5p promotes the gastric cancer development [21]. Liang et al. found that the TOB1 overexpression induced by the miR-32-5p decrease could prohibit the migration of the colorectal cancer cell, as well as its invasion [22]. In diverse cancers, miR-32-5p has been observed with variation roles, however, few investigations have considered miR-32-5p's efficacy in treating NB metastasis.

As a member of ATPases, vacuolar protein sorting 4B (VPS4B) has two isoforms (VPS4A and VPS4B) and is involved in various cellular activities $[23,24]$. Recent reports suggested that aberrant expression of VPS4B is observed in many cancer types. For instance, VPS4B functions as a tumor suppressor through inhibiting the EGFR pathway activation in breast cancer [25]. In addition, VPS4B is downregulated in breast cancer by EGFR degradation in lysosomes [26]. VPS4B is overexpressed in non-small cell lung cancer (NSCLC), and downregulation of VPS4B can inhibit cell cycle progression [27]. Several reports indicated that cell cycle arrest could be improved by VPS4B downregulation to inhibit the cell growth of hepatocellular carcinoma [28]. In addition, different expression patterns of VPS4B were found in multiple myeloma [29]. Recently, it was found that somatic copy number alterations play an essential role in malignant transformation [30]. A related review showed that approximately $30 \%$ morbidity of chromosomal 18q deletions was at the VPS4B locus. In the phase of tumorigenesis, the occurrence of VPS4B downregulation is early, and this downregulation experiences a positive selection during the primary colorectal cancer development [31].

In this investigation, NB tissues showed a lower number of miR-32-5p than the normal group. Overexpressing and inhibiting miR-32-5p mitigated and aggravated the migration of cancer and its invasion, respectively. Additionally, it was shown that miR-32-5p directly targets VPS4B, which is responsible for NB metastasis. These outcomes implicated that knocking down miR-32-5p caused NB metastasis by regulating VPS4B expression. We inferred that miR-32-5p possibly functions as a new curativetarget for NB metastasis. Furthermore, it was found that DHA synergistically promotes miR-32$5 p$ 's therapeutic effects on NB metastasis in vivo and in vitro. Therefore, the current research implicated that miR-32-5p and DHA can be considered as a target for therapeutic intervention in NB cell metastasis.

\section{Materials And Methods}

\section{Identify differentially expressed genes (DEGs) from the gene expression omnibus database}

Gene expression omnibus (GEO) (https://www.ncbi.nlm.nih.gov/geo/) was adopted to select GSE128004 and GSE121513 datasets. The GSE128004 dataset is the exosomal data of neuroblastoma cancer 
patients, including 15 patients and 3 normal controls. In the GSE121513 dataset, the number of embryonal stem cell lines, normal fetal adrenal cortex samples, normal fetal adrenal neuroblast samples, and neuroblastoma samples were 8, 7, 7, and 95, respectively. The R language package (Deseq2 package) was used to screen the DEGs in tissue samples, with adjusted $\mathrm{P}<0.01$ and $\left|\log _{2} \mathrm{FC}\right|>2$ as the threshold.

\section{Cell lineage and culture}

Cell lines of NB in human, including IMR-32, SK-N-SH, SK-N-BE and SH-SY5Y, were obtained from the National Collection of Authenticated Cell Cultures (Shanghai, China) Dulbecco's modified Eagle's medium (DMEM; HyClone, Life Sciences, Shanghai, China) was utilized to culture these cell lines, supplemented with $10 \%$ FBS (BI, Kibbutz, Israel), penicillin G (100 units; Beyotime, Beijing, China), and streptomycin (100 units, HyClone). The medium was placed in a $37^{\circ} \mathrm{C} 5 \% \mathrm{CO}_{2}$ incubator.

\section{Extraction of RNA and real-time quantitative polymerase chain reaction (qRT-PCR) analysis}

We extracted total RNA from cells and tissues using TRIzol reagent (Invitrogen, Carlsbad, California, USA) based on the manufacturer's recommendations. Reverse Transcription Kit (Fermentas, MA, USA) was adopted to synthesize complementary DNA (cDNA) from each $2 \mu \mathrm{g}$ RNA sample. SYBR premix PCR kit (Thermo) was utilized to conduct qRT-PCR on a qTOWER real-time PCR instrument (Jena, Germany). The primer sequences are listed below. The miRNA expression level was measured by the comparative threshold cycle value, and the relative change was computed by the $2^{-\Delta \Delta C t}$ method with the normalization of the target gene to that of $U 6$ expression. miR-32-5p forward primer: CGCGCGTATTGCACATTACTAA and reverse primer: AGTGCAGGGTCCGAGGTATT. The forward and reverse primers of U6 are as follows: CTCGCTTCGGCAGCACA and AACGCTTCACGAATTTGCGT, respectively.

\section{Western blotting}

Western blotting was carried out in tissues and cultured cells. Cells were harvested after drug treatment, and phosphate-buffered saline (PBS) was used for wash. RIPA lysis buffer containing protein inhibitor (Roche, CA, USA) was used to isolate total proteins, and protein samples were quantified by BCA kit (ThermoFisher) via spectrophotometer at $562 \mathrm{~nm}$. Twenty microgram protein was separated on SDSPAGE gel. After half-an-hour blocking of 5\% BSA at ambient temperature, primary antibodies were adopted to incubate blots: VPS4B (Ab224736, Abcam, 1:1000), EGFR (\#4267, CST, 1:1000), Snail (\#3879, CST, 1:1000), MMP2 (Ab97779, Abcam, 1:1000), MMP9 (Ab228402, Abcam, 1:1000), E-cadherin (\#14472, CST, 1:1000), and GAPDH (\#5174, CST, 1:1000). After overnight incubation at 4 degrees Celsius, we rinsed the membrane in TBST thrice to avoid the unbound primary antibodies. Then, a secondary antibody of goat anti-rabbit IgG H\&L (HRP) was utilized to incubate the membrane at ambient temperature for $2 \mathrm{~h}$, which was then washed by TBST. The protein bands were detected using the imaging system (Protein Simple, USA).

\section{Plasmid construction and transfection}


miR-32-5p mimic/inhibitor as well as the negative control (NC) were provided by GenePharma company (Shanghai, China). A 6-well plate was utilized to subculture cells a day before transfection. Utilizing Lipofectamine ${ }^{\circledR} 2000$ reagent (Invitrogen; Thermo Fisher Scientific Inc.), the cells of SK-N-SH were mock transfected (mimic NC, $100 \mathrm{pmol}$ ) or subjected to transfection with miR-32-5p mimic (100 pmol), and IMR-32 cells were mock transfected (inhibitor NC, 100 pmol) or transfected with miR-32-5p inhibitor (100 pmol) at 70-80\% confluency. For VPS4B expression vector construction, the full-length CDNA encoding VPS4B was PCR amplified with primers (F: 5'-CCCAAGCTTATGTCATCCACTTCGCCCAAC-3' R: 5'CGGAATTCTTAGCCTTCTTGACCAAAATCTTC-3'). VPS4B was ligated to the appropriate sites of the pcDNA3.1 vector to generate the recombinant expression vector pcDNA3.1-VPS4B, which was propagated in E. coli strain DH5a. DNA sequence analysis was used to confirm the VPS4B cDNA sequence. After that, the identified pcDNA3.1-VPS4B plasmid was utilized to transfect SK-N-SH cells.

\section{Wound healing analysis}

Cells $\left(1 \times 10^{5} / 500 \mu \mathrm{L}\right.$ per well) were placed in a plate containing 12 wells and cultured into a confluent monolayer. A line was scrapped on the adherent cells with a sterile pipette tip of $10 \mu \mathrm{L}$, washed, and incubated with $2 \%$ FBS DMEM. The cells were traced and photographed after 0,12 and $24 \mathrm{~h}$, respectively. Software of Image J (http://rsbweb.nih.gov/ij/) was used to compute the scratch width.

\subsection{Transwell assay}

Transwell chambers (Costar, MA, USA) were adopted to study the cell migration as well as its invasion with or without Matrigel (Corning, Kennebunk, ME, USA). We placed $700 \mathrm{~mL}$ medium which contained $10 \%$ FBS in the lower chamber; $3 \times 10^{4}$ cells suspended in $300 \mathrm{~mL}$ medium which contained $1 \%$ FBS were seeded into the upper chamber for 24 and $48 \mathrm{~h}$, respectively. We fixed the migrated or invaded cells across the membrane for $1 / 3 \mathrm{~h}$ and stained them for $1 / 4 \mathrm{~h}$. Finally, the cells were counted and images were collected by an Olympus BX51 microscope.

\section{Luciferase assay}

TargetScanHuman (release 7.1) and miRDB were used to predict miR-32-5p's complementary sequence at the 3'-UTR of VPS4B. Utilizing Dual luciferase 3'-UTR reporter gene detection to verify VPS4B as miR-32$5 p$ 's direct target. To construct the luciferase plasmid, the 3'-UTR sequence fragment with or without putative binding sites was inserted into pGL3 vectors (Promega, Madison, WI, USA), defined as wild-type (wt) and mutant regions. The wt plasmid of VPS4B 3'-UTR and VPS4B 3'-UTR mutant were constructed. The wt sequence of VPS4B was 5'-ACTGATACCTTTCACTGTGCAATC-3' and mut sequence was 5'CTGATACCTTTCACTACATGCACTC-3'. SK-N-SH cells $\left(5 \times 10^{5} /\right.$ well) were planted in plates of 6 wells and cultured for $24 \mathrm{~h}$ and co-transfected with Wt-VPS4B 3'-UTR or Mut-VPS4B 3'-UTR and miRNA-32-5p mimic/mimic NC using Lipofectamine 2000 (Thermo Fisher Scientific, Inc.). Two days after posttransfection, we harvested and lysed the cells. The activities of the Firefly and Renilla luciferases were measured to detect the luciferase reporter gene from cell lysates (Promega). Finally, the relative activity of 
Firefly luciferase to that of Renilla luciferase was normalized, and the fold-changes of the reporters were computed below: RLU Firefly luciferase/RLU Renilla luciferase× $100 \%$.

\section{Metastasis in nude mice}

Nude mice at the age of four to six weeks, obtained from Beijing Vital River Laboratory Animal Technology Co., Ltd., were kept under conditions of normal room temperature and humidity. Mice were given free access to conventional food as well as water. All of the animal procedures were carried out under the manuals for the care and use of experimental animals and under the approval of the IACUC of the China Academy of Chinese Medical Science. NB metastases were produced via tail vein injection of IMR-32 cells $\left(100 \mathrm{~mL}, 2 \times 10^{6}\right)$ into the nude mice. 1 week after injection, we divided the mice into the normal saline control group $(n=5)$ and two treatment groups: DHA group (100 mg/kg, $n=5)$, DHA plus mimic group (DHA $100 \mathrm{mg} / \mathrm{kg}+$ mimic $0.5 \mathrm{OD} /$ day, $\mathrm{n}=5$ ). Mice were injected DHA and miR-32-5p mimic intraperitoneally daily for 3 weeks. Subsequently, all mice were killed after anesthesia by chloral hydrate, and the lung nodules were counted and photographed. After euthanasia, we recovered the tumor, whose wet weight for each was weighed. The xenograft tumors were surgically cut and fixed for hematoxylin and eosin /immunohistochemistry (IHC) staining. In addition, the EMT protein expression was detected using the tumor tissues by Western blotting.

\section{IHC analysis}

Tumor tissue samples were fixed and embedded in paraffin. To retrieve antigens, citrate buffer $(\mathrm{pH} 6.0)$ was used in the heat process of tissue slides. Then, the slides were stained using 3,3'-diaminobenzidine (DAB) (Phoenix Biotechnologies), and Meyer's hematoxylin was utilized for counterstaining. Afterwards, we dehydrated them in ethanol. The slides were observed using a fluorescent microscope.

\section{Statistical methods}

Mean \pm standard deviation (SD) was utilized to express all data. We analyzed the comparison between groups of two using student's t-test, and explored the comparison among various groups using one-way analysis of variance. Values of $\mathrm{P}<0.05\left({ }^{\star} \mathrm{P}<0.05,{ }^{\star \star} \mathrm{P}<0.01,{ }^{\star \star *} \mathrm{P}<0.001\right)$ were markers of statistical significance.

\section{Results}

\section{miR-32-5p is downregulated in neuroblastoma cancer}

To date, the GEO database contains the maximum comprehensive public microarray/sequencing data resources. The miRNA expression profile data of tissues from patients with NB and normal controls were downloaded from GEO (GSE128004 and GSE121513), and the miR-32-5p expression in both groups was compared. The miR-32-5p expression data in GSE128004 and GSE121513 displayed that miR-32-5p was obviously lower in NB tumor samples than the healthy samples (Fig. 1A and 1B, $P<0.05$ and $P<0.001$ ). To 
investigate miR-32-5p's influence on NB cell metastasis, the miR-32-5p expression levels in the cell lines of NB cancer (IMR-32, SH-SY5Y, SK-N-SH, and SK-N-BE) were first analyzed. As illustrated in Fig. 1C, the expression level of miR-32-5p was reduced compared to 293T.

\section{miR-32-5p suppresses the NB cell migration and its invasion}

To emphatically study miR-32-5p's influence on the NB cell migration as well as its invasion, we transfected miR-32-5p mimic/inhibitor into SK-N-SH and IMR-32 cells, respectively, and the transfection efficiency was detected. Fig. 2A and 2B illustrate that the miR-32-5p expression in NB cancer cells was remarkably augmented after the transfection of miR-32-5p mimic $(P<0.01)$ and obviously lowered after the transfection of miR-32-5p inhibitor $(P<0.001)$. The migration capacity of NB cells was identified by wound healing analysis in the cells after miR-32-5p mimic/inhibitor transfection. As presented in Fig. 2C and 2D, the area and width (91.57 \pm 0.92$)$ of the scratches were obviously larger in SK-N-SH after mimic transfection than those in the control group $(85.33 \pm 0.58)$ after $12 \mathrm{~h}$, indicating that the miR-32-5p upregulation could inhibit SK-N-SH cell's migration ability. Similarly, the mi-32-5p downregulation was found to enhance IMR-32 cell's migration ability. The data suggested that the miR-32-5p upregulation significantly reduced wound closure in NB cells. Conversely, the mi-32-5p downregulation promoted the wound closure in NB cells. To further make sure if miR-32-5p affected the NB cell migration and its invasion, transwell assay was conducted to identify the changes in cell migration as well as its invasion after miR-32-5p mimic/inhibitor transfection. As shown in Fig. 2E and 2F, the upregulation of miR-32-5p remarkably reduced the number of migrated and invaded cells than that of the NC group in SK-N-SH. Contrastingly, silencing of miR-32-5p reversed this effect. Since E-cadherin, Snail, EGFR, MMP2, and MMP9 are identified as epithelial-mesenchymal transition (EMT) biomarkers, Western blotting analysis is selected to identify the protein expression. As displayed in Fig. $\mathbf{2} \mathbf{G}$ and $\mathbf{2 H}$, E-cadherin protein expression was induced, and miR-32-5p mimic restrained that of Snail, EGFR, MMP2, and MMP9, while miR-32-5p inhibitor showed an opposite effects. The original blots of Fig. $\mathbf{2 G}$ and $\mathbf{2 H}$ are presented in supplementary Fig. S1 and S2, respectively. All things considered, the assumption that miR-32-5p could inhibit the NB cell migration as well as its invasion is verified.

\section{VPS4B is miR-32-5p's direct target}

According to previous studies, the targeting gene is combined with miRNA for an effective biological function. Therefore, to explain the mechanism of miR-32-5p in NB cells, TargetScan

(www.targetscan.org/) as well as miRDB (http://mirdb.org/) was utilized to predict miR-32-5p's targets. VPS4B target which belonged to cancer metastasis gene ontology (GO) terms was identified, which is not yet reported in NB. First, we detected the mRNA level and protein level of VPS4B in NB cells (Fig. 3A and 3B) and found that VPS4B was upregulated compared to 293T, indicating an opposite trend of miR-32-5p expression as displayed in Fig. 1C. The original blots of Fig. 3B are presented in supplementary Fig. S3. qRT-PCR as well as Western blotting was adopted to detect the mRNA level and protein level of VPS4B, respectively. The results showed that these two levels were decreased in cells of SK-N-SH transfected with miR-32-5p mimic ( $P<0.05 ;$ Fig. 3C and 3D), while the miR-32-5p inhibition improved these levels of VPS4B 
in IMR-32 cells ( $P<0.05$; Fig. 3E and 3F). The original blots of Fig. 3D and 3F are presented in supplementary Fig. S3. The findings implicated that VPS4B and miR-32-5p in NB cells were negatively related. Moreover, we cloned wt or mutated 3'-UTR of VPS4B into firefly luciferase reporters to validate whether VPS4B is miR-32-5p's direct target (Fig. 3G); the dual-luciferase reporter gene assay illustrated that the signal of luciferase was inhibited after cotransfection with miR-32-5p and VPS4B wild-type 3'UTR luciferase reporter, rather than the mutant UTR (Fig. 3H), suggesting that miR-32-5p controls VPS4B expression via the direct binding to the $3^{\prime}-\mathrm{UTR}$.

\section{VPS4B is necessary for miR-32-5p's regulatory function on NB cell metastasis}

To further evaluate whether VPS4B could contribute to biological effects of miR-32-5p, VPS4B was overexpressed by transfecting plasmid into cells of SK-N-SH, with downregulated miR-32-5p (Fig. 4A and 4B). The original blots of Fig. 4B are presented in supplementary Fig. S4.

The overexpression of VPS4B resulted in significant migration and invasion in cells of SK-N-SH compared oeVPS4B-NC with vector-NC group (Fig. 4C-G), indicating that VPS4B is a regulator of SK-N-SH cell metastasis. Afterwards, rescue experiments were conducted to explore whether VPS4B could influence miR-32-5p's functional roles in cells. We transfected cells of SK-N-SH with miR-32-5p mimic together with NC or oeVPS4B plasmid. Those results of wound-healing analysis, migration, and invasion analysis demonstrated that the co-transfection with oeVPS4B plasmid and mimic rescued the functional effects of miR-32-5p overexpression on metastasis ( $\mathrm{P}<0.05$ ) (Fig. 4C-E) of NB cancer cells. Specifically, VPS4B overexpression restored the decreased wound area (Fig. 4C) and the elevated invasion and migration cell numbers (Fig. 4D and 4E) of NB cells after miR-32-5p mimic transfection. The findings indicated that miR-32-5p targets VPS4B and affects NB cell metastasis. Western blotting analysis also revealed that cotransfection with oeVPS4B plasmid recovered the downregulation of VPS4B, EGFR, Snail, MMP2, and MMP9 protein and the upregulation of E-cadherin caused by the miR-32-5p mimic in cells of SK-N-SH $(P<0.05)$ (Fig. 4F and 4G). The original blots of Fig. 4F and 4G are presented in supplementary Fig. S4 and S5, respectively.

\section{miR-32-5p increases sensitivity to dihydroartemisinin in neuroblastoma cancer cells}

Since both miR-32-5p and DHA affect NB cancer metastasis based on the results above and other studies, we explored whether miR-32-5p increases sensitivity to DHA in NB cancer cells after miR-32-5p mimic/inhibitor transfection and then treated with different DHA concentrations for a day. Wound healing, transwell assays, and Western blotting inplicated that the scratches' area and width were remarkably larger in the DHA group than those in the NC group at $12 \mathrm{~h}$ and $24 \mathrm{~h}$, indicating that DHA inhibits the metastasis ability of cancer cells (Fig. 5A, upper panel). Conversely, the upregulation of miR32-5p with mimic (Fig. 5B, lower panel) could promote the suppressive effects of DHA on NB cell metastasis. Moreover, the miR-32-5p knockdown could also improve the role of DHA on NB cell metastasis at $12 \mathrm{~h}$ and $24 \mathrm{~h}$ (Fig. 5B). Furthermore, DHA markedly reduced the migrated and invaded cells in comparison with the NC group (Fig. 5C-F, upper panel), while miR-32-5p mimic synergistically inbibited the migration and invasion capacities of cells with DHA cotreatment (Fig. 5C and 5E, lower 
panel). Additionally, the miR-32-5p inhibitor improves DHA's role on NB cell metastasis (Fig. 5D and 5F, lower panel). Western blotting implicated that DHA decreased the expression of EGFR, MMP2, MMP9, and Snail and increased E-cadherin without miR-32-5p mimic, while DHA drastically strengthened the effects above with miR-32-5p mimic (Fig. 5G). The original blots of Fig. 5G are presented in supplementary Fig. S6. Interestingly, miR-32-5p inhibitor restored the expression variation of these EMT proteins induced by DHA treatment (Fig. $\mathbf{5 H}$ ). The results verified that miR-32-5p promotes DHA's suppressive effect on NB cell metastasis. The original blots of Fig. $\mathbf{5 H}$ are presented in supplementary Fig. S7.

\section{VPS4B is involved in the cotreatment roles of miR-32-5b mimic and DHA on NB cell metastasis}

To further explore the role of VPS4B in miR-32-5p and DHA's biological effects, we first detected its expression in cells of SK-N-SH and found that miR-32-5p mimic and DHA dramatically downregulates VPS4B, while miR-32-5p inhibitor and DHA upregulates VPS4B (Fig. 6A and 6B). The original blots of Fig. 6A and $6 \mathrm{~B}$ are presented in supplementary Fig. S8. Then, we analyzed whether the VPS4B enhancement could rescue the NB metastasis inhibitory effect of miR-32-5p mimic and DHA in SK-N-SH cells. Western blotting showed that the VPS4B overexpression in cells with mimic after DHA therapy rescued VPS4B and EMT protein expression levels (Fig. 6C and 6D). The original blots of Fig. $6 \mathrm{C}$ and $6 \mathrm{D}$ are presented in supplementary Fig. S4 and S5, respectively. Similarly, the wound healing analysis showed that the wound area was larger in the DHA-oeVPS4B-mimic group than the DHA-oeVPS4B-NC group (Fig. 6E). Additionally, transwell analysis indicated that VPS4B overexpression had a reverse effect on the inhibited cell migration as well as its invasion by DHA and miR-32-5p mimic (Fig. 6F and 6G). The results indicated that VPS4B overexpression significantly attenuates miR-32-5p and DHA's suppressive effect on metastasis, further making sure that the suppressive role of miR-32-5p and DHA on the NB cell metastasis was targeted by VPS4B.

\section{DHA and miR-32-5p abrogate neuroblastoma metastasis in a xenograft model of nude mice}

To directly assess miR-32-5p and DHA's role in the metastasis model in vivo, a xenograft metastasis NB model in nude mice was established. SK-N-SH cells with overexpressed miR-32-5p were established, and the overexpression efficiency was confirmed (Fig. 7A). A similar in vitro study revealed that miR-32-5p's target gene VPS4B was downregulated in the nude mouse model (Fig. 7B). Protein-level VPS4B expression was analyzed by Western blotting and immunofluorescent staining, indicating decreased VPS4B expression, which confirmed the results above (Fig. 7C and 7D). Subsequently, SK-N-SH cells were inoculated into the nude mice by tail vein injection with/without miR-32-5p mimic and DHA treatment. After sacrificing the mice, lung tissues were collected and metastasis nodes counted. The number of metastatic pulmonary nodules was sharply reduced in the mimic group than that in the NC group, and DHA cotreatment with miR-32-5p mimic suppressed more than the mimic group (Fig. 7E). These tumors were confirmed by H\&E staining (Fig. 7F). The original blots of Fig. 7F are presented in supplementary Fig. S9. To investigate whether miR-32-5p inhibited the EMT in vivo, the expression of EGFR, Snail, MMP2, MMP9 and E-cadherin was detected. Results showed that E-cadherin was increased and EGFR, Snail, MMP2, and MMP9 were decreased in the miR-32-5p overexpression and DHA treatment groups, which 
matched well with the in vitro results (Fig. 7G), indicating that the inhibitory metastasis role was effectuated by preventing the EMT process in vivo, like the in vitro study. The original blots of Fig. 7G are presented in supplementary Fig. S9.

\section{Discussion}

Neuroblastoma (NB) cancer is one of the deadliest tumors that start in early nerve cells. NB mortality is main caused by metastasis [3]. Thus, searching for new therapies for NB metastasis is an area of current research interest. Herein, miR-32-5p was downregulated in the exosome of NB patients compared to normal controls using GEO data mining. Furthermore, VPS4B was identified as the miR-32-5p's direct target in NB cell lines. VPS4B overexpression rescued the influence of miR-32-5p upregulation on the NB cancer cell metastasis. For all we know, this is the first study to probe into the inhibitory effect of miR-32$5 p$ in NB tumor cells by targeting VPS4B.

Hitherto, miR-32-5p was claimed to be abnormally expressed in some tumor types and function as critical regulators. miR-32-5p serves as a suppresser of tumor in several cancers, either inhibiting proliferation or metastasis, including cervical cancer [32], clear cell renal cell carcinoma [33] as well as colorectal cancer cells [22]. However, no study has explored the involvement of miR-32-5p in NB cancer metastasis.

For cancer metastasis, EMT is considered a key sponsor of metastasis, characterized by an attenuation of interactive cell junctions and the re-establishment of the cytoskeleton. Hence, we explored miR-32-5p's effect on EMT by detecting the EMT marker expression using Western blotting. E-cadherin is one of the most commonly used markers for epithelial traits, and Snail and EGFR are the common markers for mesenchyma [34]. Matrix metalloproteinase 2 (MMP-2) and MMP-9 have been identified in large quantities in tumors and related to tumor metastasis $[35,36]$. Herein, we discovered that the overexpression of miR-32-5p remarkably prohibited EMT-associated migration and invasion by downregulation of MMP2, MMP9, Snail, and EGFR and upregulation of E-cadherin in NB cancer cells, while the downregulation of miR-32-5p had an opposite effect. Furthermore, increased miR-32-5p expression significantly suppressed tumor metastasis in vivo, which is consistent with previously reported findings by Wang et al. [33]. However, in prostate cancer [37], pancreatic cancer [38] and tuberous sclerosis complex [39], the miR-32-5p level was greater than that in the corresponding normal tissues. These contradictory reports suggested that miR-32-5p might function differently in different tumors.

miRNAs function as posttranscriptional gene regulators in cancer by targeting specific genes participating in various cell processes, including cell proliferation, apoptosis, and metastasis. Herein, we found the VPS4B expression, miR-32-5p's direct target, was controlled by miR-32-5p partially because the decreased stability of its mRNA. The upregulation of miR-32-5p remarkably lowered the VPS4B expression either at the mRNA or protein level, inhibiting NB metastasis. This phenomenon was also observed in another study, wherein the siRNA-induced VPS4B downregulation promoted cell cycle arrest and inhibited hepatocellular carcinoma progression [27]. Additionally, VPS4B overexpression significantly 
promoted neuroblastoma cell metastasis, similar to the phenotype observed following miR-32-5p inhibition, consistent with that of the previous study, which stated that VPS4B was highly expressed, affecting tumor size and clinical stage in NSCLC [28]. Therefore, in-depth investigations are required to decipher miR-32-5p's functions in NB metastasis and development by targeting the VPS4B signaling pathway. The overexpression of VPS4B reversed the effect on EMT markers caused by miR-32-5p upregulation in DHA treatment, indicating that miR-32-5p/VPS4B axis is participating in inhibiting NB metastasis. However, the mechanism analysis found that VPS4B takes a positive role in NB metastasis, which is opposite to that stated in early studies showing that VPS4B presents decreased expression in multiple myeloma cells [29] and in the phase of tumorigenesis, its downregulation happens early and experiences a positive selection during the primary colorectal cancer development [31]. This phenomenon could ascribe VPS4B increase in various types of cancer that may be explained by the utilization of various signaling pathways.

As a main active metabolite derivative of artemisinin [40], DHA has drawn the attention of researchers for its potential in the clinical treatment of diseases other than malaria, especially its activity on cancer metastasis [41]. Previous studies reported that DHA inhibits metastasis in various cancers [42-44]. Some preceding studies have shown that is more effective for therapy in combination with two or more compounds than with a single one alone [45]. Gao et al. found that cancer cell migration was inhibited by Cdc42-related signaling pathway after a combination of DHA and Resveratrol treatment [46]. In addition, the DHA and miR-29b cotreatment promoted the apoptosis on cholangiocarcinoma cells HUCCT-1 and FRH0201 [47]. The current results suggested that the DHA and miR-32-5p combination was more effective in reducing metastasis of NB cancer cells compared to DHA or miR-32-5p alone in vitro and in vivo, suggesting that the manipulation of miR-32-5p levels combined with DHA may have therapeutic potential for NB patients. Additionally, the miR-32-5p overexpression and DHA treatment decreased the expression of EGFR, Snail, MMP2, and MMP9 and promoted the expression of E-cadherin. Transwell assays were also consistent with Western blotting results above. Importantly, we showed that miR-32-5p plus DHA treatment has a significant inhibitory effect than miR-32-5p or DHA alone. Shin et al. reported that miR-32 as well as its target SLC45A3 regulated the lipid homeostasis of oligodendrocytes [31], indicating that miR-32 is closely related to neuronal activity, which could be used as a supplement for human neuroblastoma treatment.

The current findings demonstrated that miR-32-5p could target VPS4B directly and inhibit the metastasis of neuroblastoma cells with DHA. Therefore, these data might provide a novel treatment strategy, molecular-targeted therapy, which might resolve the current issues related to drug scarcity. Herein, we focused on miR-32-5p and its target VPS4B, however, it is also necessary for us to further investigate the other miR-32 members to reveal their probable roles in NB clinical progress. In addition, the small number of participants in GEO data or no clinic data in this study limited our understanding of the complex function of miR-32. Therefore, some clinical samples need to be included to verify the findings of this study in future studies. 


\section{Declarations}

\section{Acknowledgements}

The authors would like to thank all study team members for valuable discussions and their time spent on this research. The authors thank the Fundamental Research Funds for the Central public welfare research institutes (grant number ZZ13-YQ-100), the National Natural Science Foundation of China (grant numbers: 81803814 and 81841001) and China Academy of Chinese Medical Sciences Innovation Fund

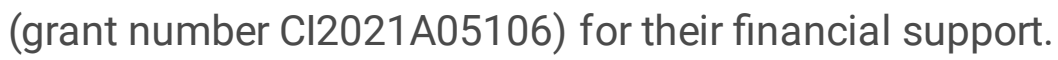

\section{Author contributions}

C.L.N., L.Y.J. and C.H.H. designed the experiment, analyzed the data, wrote and modified the manuscript. L.H., Z.Z.Y., W.X., Q.S.Q., Y.Y.M. and D.S.Q. helped to complete the animal experiment. C.L.N. carried out the western blot and transwell assays. L.H., Z.Y. and L.T. performed the qRT-PCR, IHC staining and luciferase analysis. All authors contributed to the manuscript and approved the submitted version.

\section{Statement}

We confirm the study is reported in accordance with ARRIVE (Animal Research: Reporting of In Vivo Experiments) guidelines and all methods are carried out in accordance with relevant guidelines and regulations.

\section{Data availability}

All data generated or analyzed during this study are available.

\section{Ethics approval}

The animal experiment in this study was approved by the Animal Ethics Committee of the Institute of Chinese Materia Medica, China Academy of Chinese Medical Sciences.

\section{Conflicts of interest}

Lina Chen, Liu Tuo and Yujie Li are employees of Artemisinin Research Center, Institute of Chinese Materia Medica, China Academy of Chinese Medical Sciences. All authors declare that they have no conflict of interest.

\section{References}

1. Vinklarek, J., Novak, J., Bienertova-Vasku, J., Sterba, J., and Slaby, O., The role of MicroRNAs in the pathophysiology of neuroblastoma and their possible use in diagnosis, prognosis and therapy. Klin. Onkol. 27, 331-9 (2014). 
2. Park, J.R., Eggert, A., and Caron, H., Neuroblastoma: biology, prognosis, and treatment. Hematol Oncol. Clin. North Am. 24, 65-86 (2010).

3. Mahapatra S. and Challagundla K.B., Neuroblastoma, (StatPearls Publishing [Internet], Treasure Island, Florida, USA 2019), Available from: https://www.ncbi.nlm.nih.gov/books/NBK448111/.

4. Ahmed, A.A., Zhang, L., Reddivalla, N., and Hetherington, M., Neuroblastoma in children: Update on clinicopathologic and genetic prognostic factors. Pediatr. Hematol. Oncol. 34, 165-185 (2017).

5. Zhou, X., et al., MicroRNA-429 inhibits neuroblastoma cell proliferation, migration and invasion via the NF-kappaB pathway. Cell Mol. Biol. Lett. 25, 5 (2020).

6. Efferth, T., From ancient herb to modern drug: Artemisia annua and artemisinin for cancer therapy. Semin Cancer Biol. 46, 65-83 (2017).

7. Gao, P., et al., Dihydroartemisinin Inhibits the Proliferation of Leukemia Cells K562 by Suppressing PKM2 and GLUT1 Mediated Aerobic Glycolysis. Drug Des. Devel. Ther. 14, 2091-2100 (2020).

8. Lin, R., et al., Dihydroartemisinin (DHA) induces ferroptosis and causes cell cycle arrest in head and neck carcinoma cells. Cancer Lett. 381, 165-75 (2016).

9. Li, Y., et al., Dihydroartemisinin inhibits the tumorigenesis and metastasis of breast cancer via downregulating CIZ1 expression associated with TGF-beta1 signaling. Life Sci. 248, 117454 (2020).

10. Li, Y., et al., Dihydroartemisinin Increases the Sensitivity of Photodynamic Therapy Via NFkappaB/HIF-1alpha/VEGF Pathway in Esophageal Cancer Cell in vitro and in vivo. Cell Physiol. Biochem. 48, 2035-2045 (2018).

11. Du, J., et al., DHA inhibits proliferation and induces ferroptosis of leukemia cells through autophagy dependent degradation of ferritin. Free Radic. Biol. Med. 131, 356-369 (2019).

12. Wu, L., Cheng, Y., Deng, J., Tao, W., and Ye, J., Dihydroartemisinin Inhibits Proliferation and Induces Apoptosis of Human Hepatocellular Carcinoma Cell by Upregulating Tumor Necrosis Factor via JNK/NF-kappaB Pathways. Evid. Based Complement Alternat. Med. 2019, 9581327 (2019).

13. Qu, C., et al., Dihydroartemisinin Exerts Anti-Tumor Activity by Inducing Mitochondrion and Endoplasmic Reticulum Apoptosis and Autophagic Cell Death in Human Glioblastoma Cells. Front. Cell Neurosci. 11, 310 (2017).

14. Huang, L., et al., An artemisinin-mediated ROS evolving and dual protease light-up nanocapsule for real-time imaging of lysosomal tumor cell death. Biosens. Bioelectron. 92, 724-732 (2017).

15. Li, Y., Zhou, X., Liu, J., Yuan, X., and He, Q., Therapeutic Potentials and Mechanisms of Artemisinin and its Derivatives for Tumorigenesis and Metastasis. Anticancer Agents Med. Chem. 20, 520-535 (2020).

16. Galardi, A., et al., MicroRNAs in Neuroblastoma: Biomarkers with Therapeutic Potential. Curr. Med. Chem. 25, 584-600 (2018).

17. Chen, E., et al., MiR-32 promotes tumorigenesis of colorectal cancer by targeting BMP5. Biomed. Pharmacother. 106, 1046-1051 (2018). 
18. Lai, E.C., Tomancak, P., Williams, R.W., and Rubin, G.M., Computational identification of Drosophila microRNA genes. Genome Biol. 4, R42 (2003).

19. Bartel, D.P., MicroRNAs: genomics, biogenesis, mechanism, and function. Cell. 116, 281-97 (2004).

20. Yan, T., et al., miR-32-5p-mediated Dusp5 downregulation contributes to neuropathic pain. Biochem. Biophys. Res. Commun. 495, 506-511 (2018).

21. Wang, Q., et al., microRNA-32-5p targets KLF2 to promote gastric cancer by activating PI3K/AKT signaling pathway. Am. J. Transl. Res. 11, 4895-4908 (2019).

22. Liang, H., Tang, Y., Zhang, H., and Zhang, C., MiR-32-5p Regulates Radiosensitization, Migration And Invasion Of Colorectal Cancer Cells By Targeting TOB1 Gene. Onco. Targets Ther. 12, 9651-9661 (2019).

23. Scheuring, S., et al., Mammalian cells express two VPS4 proteins both of which are involved in intracellular protein trafficking. J. Mol. Biol. 312, 469-80 (2001).

24. Xu, L., Zhai, L., Ge, Q., Liu, Z., and Tao, R., Vacuolar Protein Sorting 4B (VPS4B) Regulates Apoptosis of Chondrocytes via p38 Mitogen-Activated Protein Kinases (MAPK) in Osteoarthritis. Inflammation. 40, 1924-1932 (2017).

25. Lin, H.H., et al., Identification of an AAA ATPase VPS4B-dependent pathway that modulates epidermal growth factor receptor abundance and signaling during hypoxia. Mol. Cell Biol. 32, 112438 (2012).

26. Liao, Z., et al., An Internal Standard-Assisted Synthesis and Degradation Proteomic Approach Reveals the Potential Linkage between VPS4B Depletion and Activation of Fatty Acid beta-Oxidation in Breast Cancer Cells. Int. J. Proteomics. 2013, 291415 (2013).

27. Liu, Y., et al., Vacuolar protein sorting 4B, an ATPase protein positively regulates the progression of NSCLC via promoting cell division. Mol. Cell Biochem. 381, 163-71 (2013).

28. Jiang, D., et al., High expression of vacuolar protein sorting 4B (VPS4B) is associated with accelerated cell proliferation and poor prognosis in human hepatocellular carcinoma. Pathol. Res. Pract. 211, 240-7 (2015).

29. Tang, J., et al., Cell adhesion down-regulates the expression of vacuolar protein sorting 4B (VPS4B) and contributes to drug resistance in multiple myeloma cells. Int. J. Hematol. 102, 25-34 (2015).

30. Beroukhim, R., et al., The landscape of somatic copy-number alteration across human cancers. Nature. 463, 899-905 (2010).

31. Szymanska, E., et al., Synthetic lethality between VPS4A and VPS4B triggers an inflammatory response in colorectal cancer. EMBO Mol. Med. 12, e10812 (2020).

32. Liu, Y.J., et al., MiR-32-5p regulates the proliferation and metastasis of cervical cancer cells by targeting HOXB8. Eur. Rev. Med. Pharmacol. Sci. 23, 87-95 (2019).

33. Wang, M., et al., Preclinical studies using miR-32-5p to suppress clear cell renal cell carcinoma metastasis via altering the miR-32-5p/TR4/HGF/Met signaling. Int. J. Cancer. 143, 100-112 (2018). 
34. Busch, E.L., et al., Evaluating markers of epithelial-mesenchymal transition to identify cancer patients at risk for metastatic disease. Clin. Exp. Metastasis. 33, 53-62 (2016).

35. Tucker, G.C., Boyer, B., Gavrilovic, J., Emonard, H., and Thiery, J.P., Collagen-mediated dispersion of NBT-II rat bladder carcinoma cells. Cancer Res. 50, 129-37 (1990).

36. Lochter, A., et al., Matrix metalloproteinase stromelysin-1 triggers a cascade of molecular alterations that leads to stable epithelial-to-mesenchymal conversion and a premalignant phenotype in mammary epithelial cells. J. Cell Biol. 139, 1861-72 (1997).

37. Paziewska, A., et al., Candidate diagnostic miRNAs that can detect cancer in prostate biopsy. Prostate. 78, 178-185 (2018).

38. Gao, Z.Q., et al., Long non-coding RNA GAS5 suppresses pancreatic cancer metastasis through modulating miR-32-5p/PTEN axis. Cell Biosci. 7, 66 (2017).

39. Bagla, S., et al., A distinct microRNA expression profile is associated with alpha[(11)C]-methyl-Ltryptophan (AMT) PET uptake in epileptogenic cortical tubers resected from patients with tuberous sclerosis complex. Neurobiol. Dis. 109, 76-87 (2018).

40. Huang, T.E., et al., Evaluation of the Anticancer Activity of a Bile Acid-Dihydroartemisinin Hybrid Ursodeoxycholic-Dihydroartemisinin in Hepatocellular Carcinoma Cells. Front. Pharmacol. 11, 599067 (2020).

41. Que, Z., et al., Dihydroartemisin inhibits glioma invasiveness via a ROS to P53 to beta-catenin signaling. Pharmacol. Res. 119, 72-88 (2017).

42. Zhang, F., et al., Dihydroartemisinin inhibits TCTP-dependent metastasis in gallbladder cancer. J. Exp. Clin. Cancer Res. 36, 68 (2017).

43. Fei, W., et al., Targeted GSH-exhausting and hydroxyl radical self-producing manganese-silica nanomissiles for MRI guided ferroptotic cancer therapy. Nanoscale. 12, 16738-16754 (2020).

44. Pasupuleti, B.G., Khongsti, K., Das, B., and Bez, G., 1,2,3-Triazole tethered 1,2,4-trioxanes: Studies on their synthesis and effect on osteopontin expression in MDA-MB-435 breast cancer cells. Eur. J. Med. Chem. 186, 111908 (2020).

45. Mutch, D.G., Gemcitabine combination chemotherapy of ovarian cancer. Gynecol. Oncol. 90, S16-20 (2003).

46. Gao, J., Ma, F., Wang, X., and Li, G., Combination of dihydroartemisinin and resveratrol effectively inhibits cancer cell migration via regulation of the DLC1/TCTP/Cdc42 pathway. Food Funct. 11, 9573-9584 (2020).

47. Hu, H., et al., Dihydroartemisinin/miR-29b combination therapy increases the pro-apoptotic effect of dihydroartemisinin on cholangiocarcinoma cell lines by regulating Mcl-1 expression. Adv. Clin. Exp. Med. 29, 911-919 (2020).

\section{Figures}



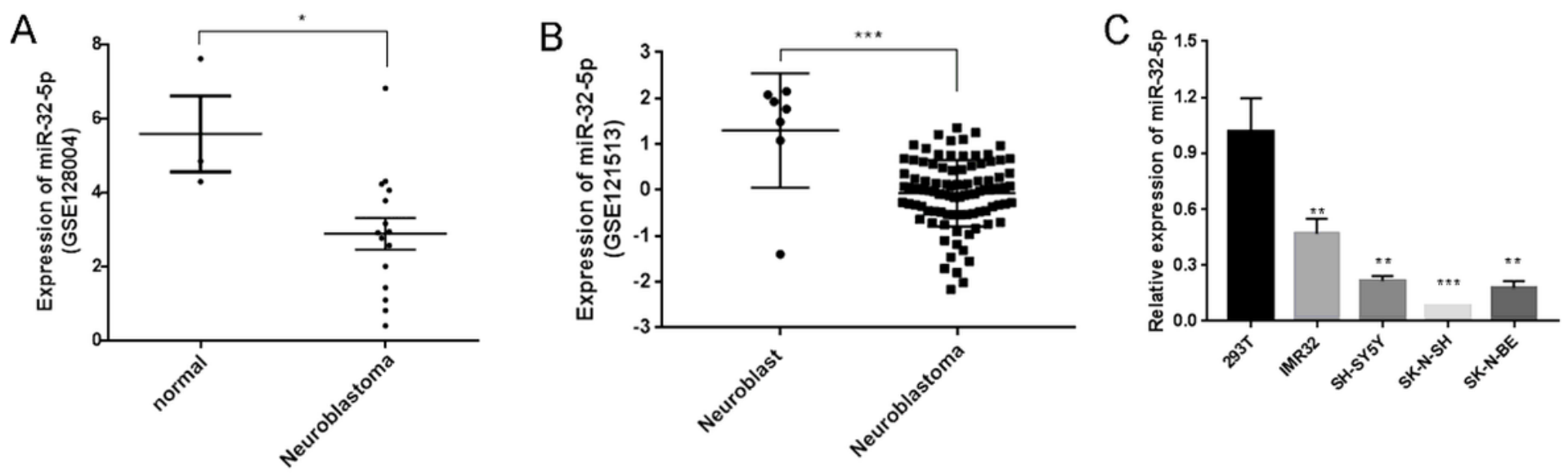

Figure 1

miR-32-5p expression in both NB cells and tissues. (A) miR-32-5p was knocked out in NB samples $(n=15)$ compared to the normal control $(n=3)$ in GSE128004. (B) miR-32-5p was knocked out in data from GSE121513 in NB specimens ( $n=95)$ compared to the normal fetal adrenal cortex samples $(n=7)$. (C) The miR-32-5p expression in cell lines of NB and HEK293T cell line was different from each other. All data are represented as mean $\pm S D$. 


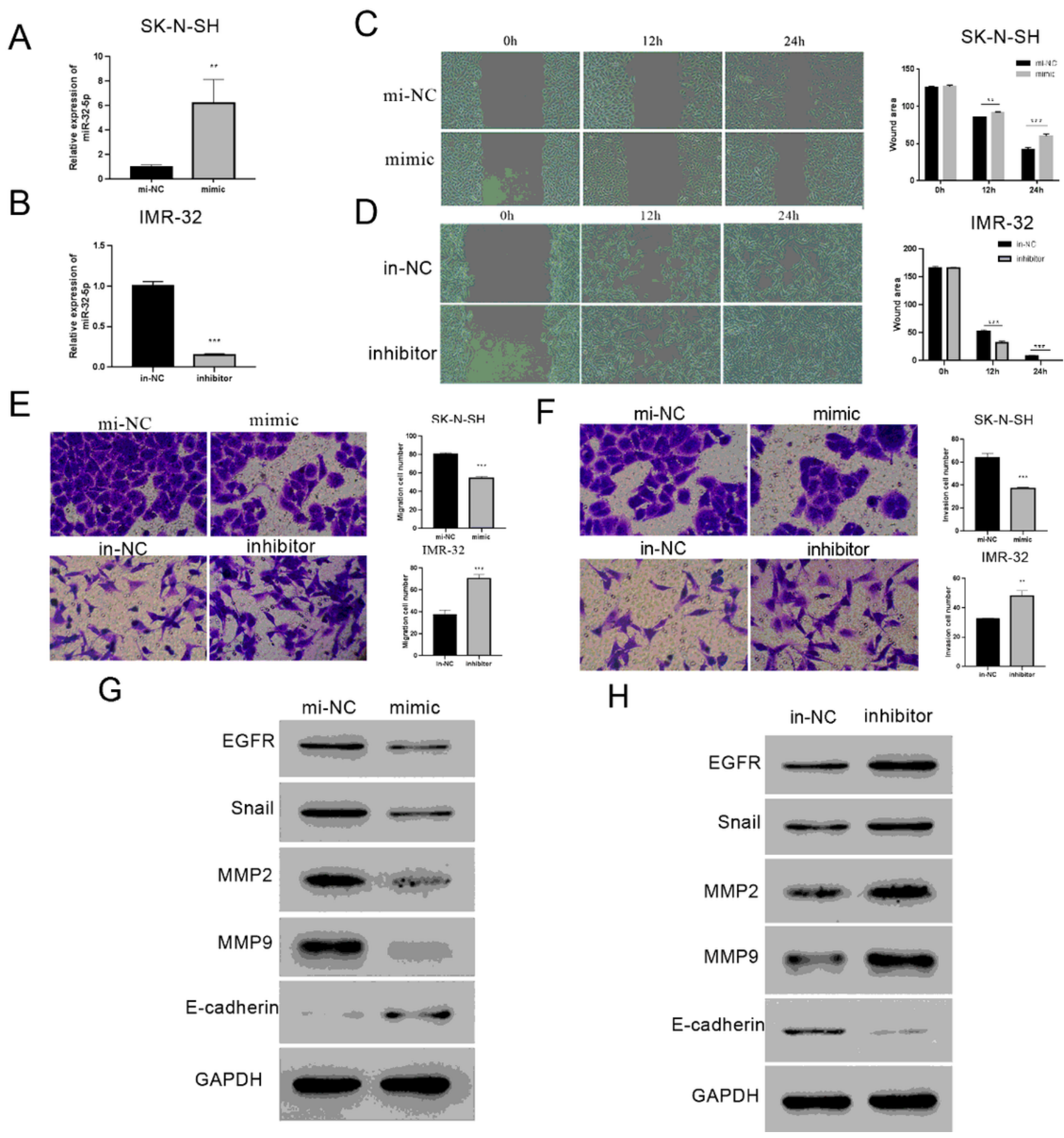

Figure 2

Influences of miR-32-5p on cell metastasis of SK-N-SH and IMR-32. Efficiency of miR-32-5p mimic in SK$\mathrm{N}-\mathrm{SH}$ (A) and inhibitor in IMR-32 (B) cells detected using qRT-PCR. Wound healing assays were achieved in cells of SK-N-SH with miR-32-5p mimic or mimic negative control (C) and IMR-32 cells with miR-32-5p inhibitor or inhibitor negative control (D). Pictures were captured at $0 \mathrm{~h}, 12 \mathrm{~h}$, and $24 \mathrm{~h}$ at $100 \mathrm{x}$ magnification. Cell migration as well as invasion was analyzed in SK-N-SH (E) or IMR-32 (F) cells after miR-32-5p mimic/inhibitor transfection by transwell assay. The cell number of SK-N-SH or IMR-32 in three stochastic microscopic fields (100x) was calculated per group. The changes in proteins associated with 
metastasis in cells of SK-N-SH (G) or IMR-32 $(\mathrm{H})$ were measured by Western blotting. Data are represented as the mean $\pm S D$. NC: negative control, mi: mimic, in: inhibitor.

A

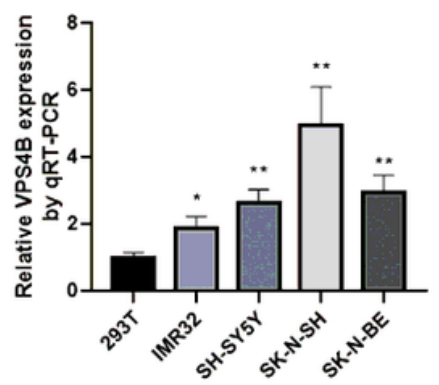

C

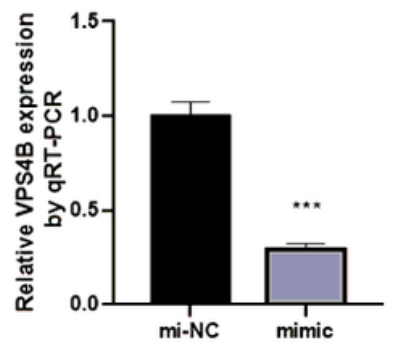

E

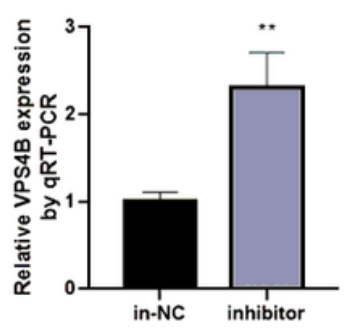

B

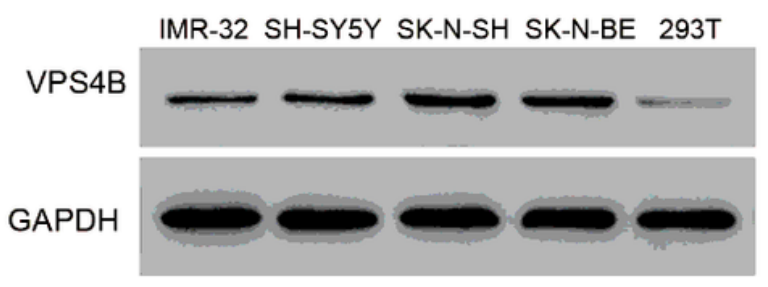

G

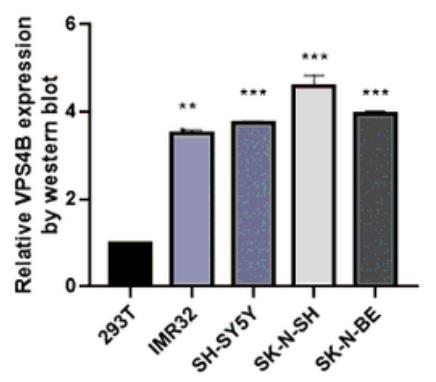

$\mathrm{D}$

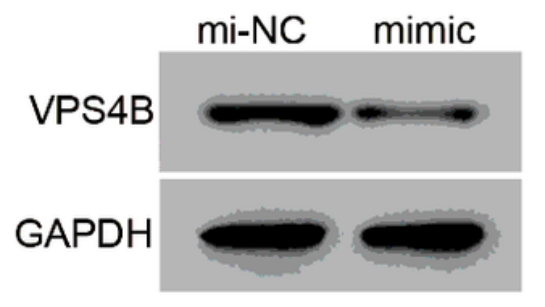

$\mathrm{F}$

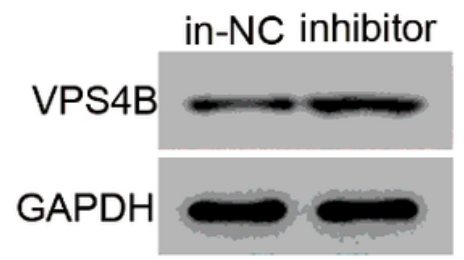

wtVPS4B 3'-UTR 5' -ACTGATACCTTTCACTGTGCAATC- 3' || || |||

hsa-miR-32-5p 3'-ACGUUGAAUCAUUACACGUUAU- 5' mutVPS4B 3'-UTR 5' - CTGATACCTTTCACTACATGCACTC- 3'

$\mathrm{H}$

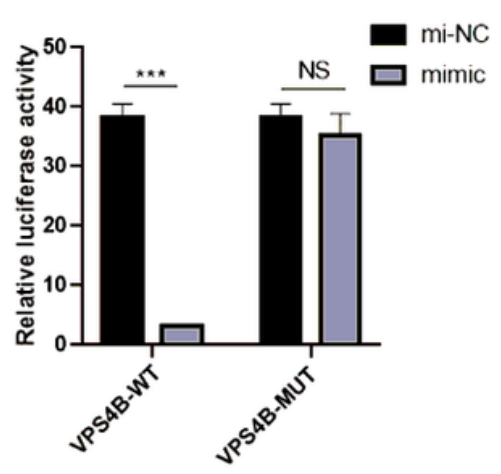

Figure 3

VPS4B 3'-UTR is miR-32-5p's target. VPS4B expression at transcript (A) and protein levels (B) in NB cells. (C) and (D) The expression of VPS4B at transcript and protein levels were measured in cells of SK-N-SH after transfection with mi-NC or miR-32-5p mimic. (E) and (F) The VPS4B expression at transcript and protein levels were measured in cells of IMR-32 after transfection with in-NC or miR-32-5p inhibitor. (G) The 3'-UTR region of VPS4B mRNA matches the miR-32-5p sequence. $(\mathrm{H})$ The activity of luciferase was detected in cell of SK-N-SH after transfection with miR-32-5p mimic and WT-VPS4B-3'-UTR or Mut-VPS4B3'-UTR plasmid. Data are shown as means $\pm S D$; NC: negative control, mi:mimic, in: inhibitor, WT-VPS4B-3'UTR: wild type-VPS4B-3'-untranslated region, Mut-VPS4B-3'-UTR: mutation-VPS4B-3'-untranslated region. 

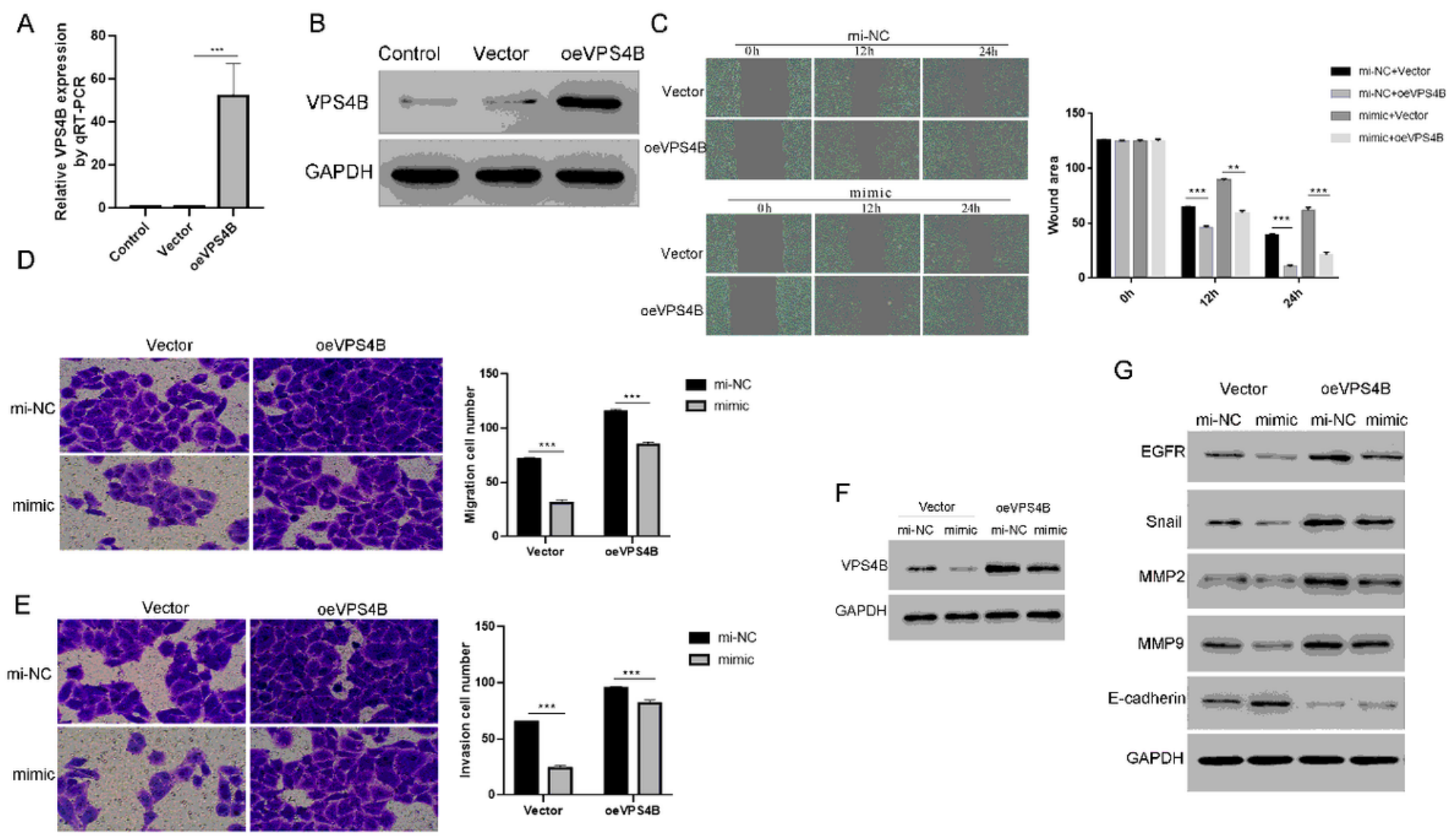

\section{Figure 4}

Overexpression of VPS4B reversed miR-32-5p-mediated effects on the cell metastasis of SK-N-SH. (A) and (B) Overexpression efficiency of VPS4B in cells of SK-N-SH was detected using qRT-PCR and Western blotting. (C) Wound healing assays were performed in cells of SK-N-SH after cotransfection with oeVPS4B plasmid and miR-32-5p mimic, and pictures were taken at $0 \mathrm{~h}, 12 \mathrm{~h}$, and $24 \mathrm{~h}$ at $100 \mathrm{x}$ magnification. The quantification of wound closure was listed on the right panel. (D) and (E) Transfecting VPS4B plasmid significantly reversed cell migration as well as its invasion of SK-N-SH cells mediated by miR-32-5p mimic treatment (100x magnification). (F) and (G) Co-transfection with oeVPS4B plasmid and miR-32-5p mimic recovered the downregulation of VPS4B, EGFR, Snail, MMP2, and MMP9 and E-cadherin upregulation caused by miR-32-5p mimic in cells of SK-N-SH. Data are represented as means \pm SD. oe VPS4B: overexpression of VPS4B, mi:mimic, NC: negative control. 

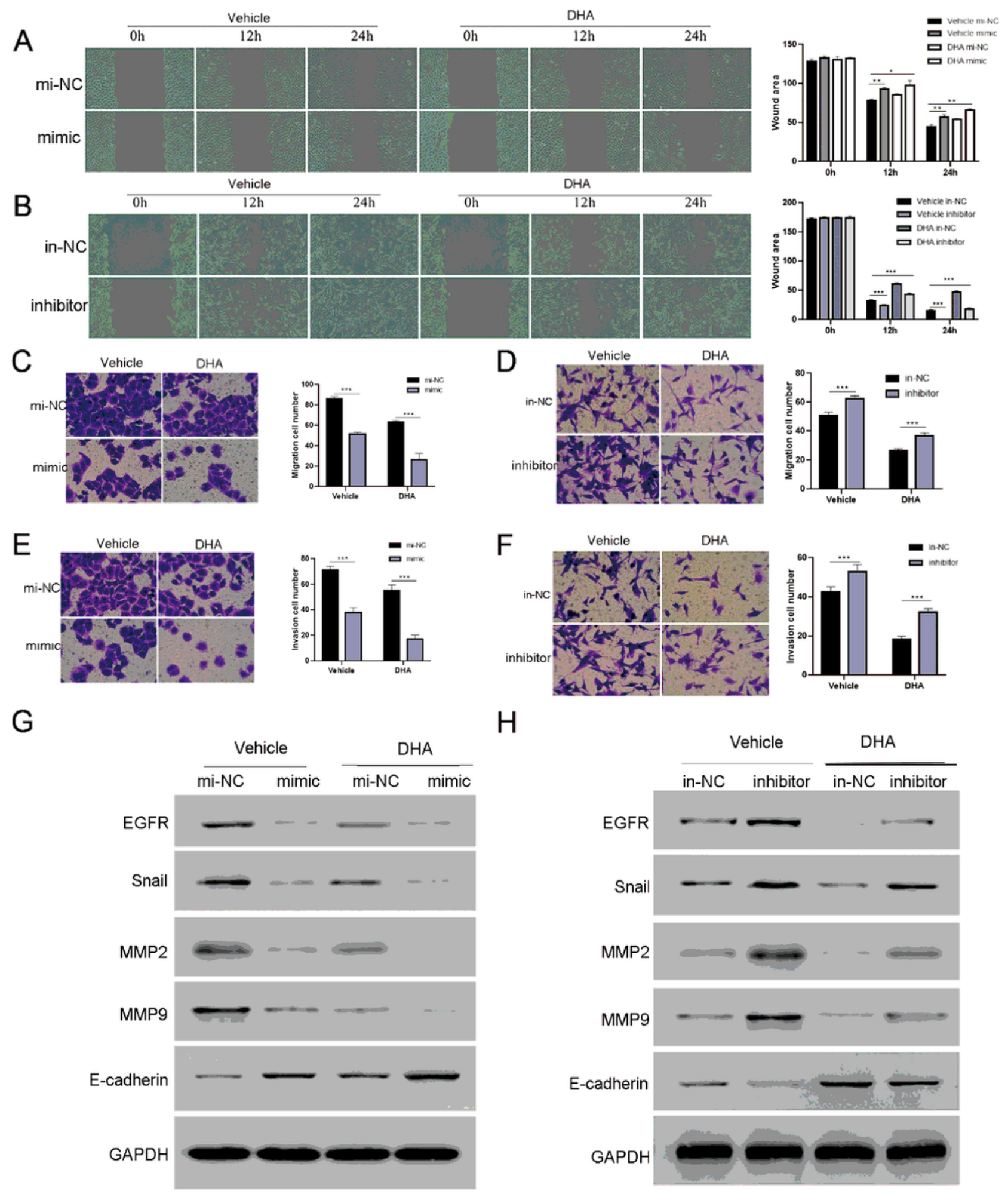

$\mathrm{H}$

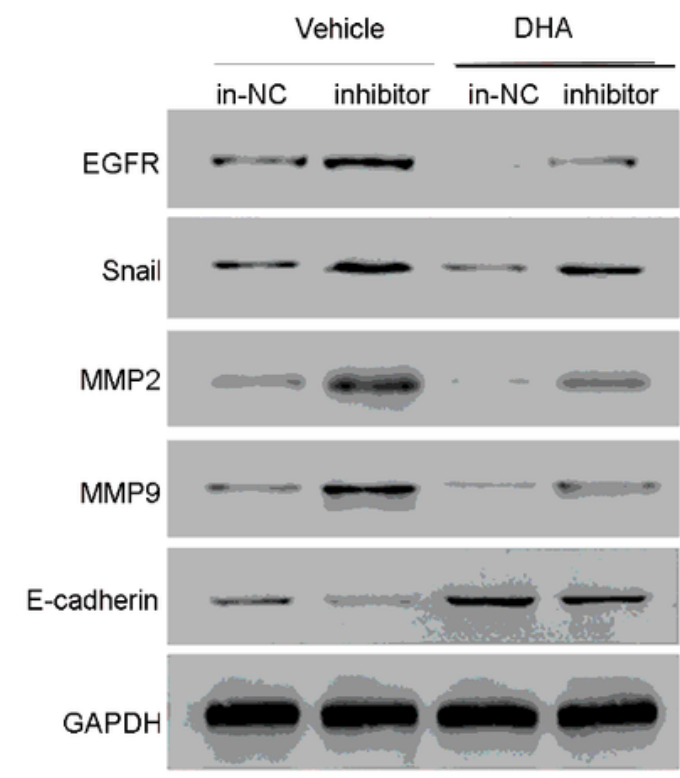

\section{Figure 5}

MicroRNA-32-5p increases the sensitivity to DHA in neuroblastoma. (A) and (B) Wound-healing assay was performed with SK-N-SH cells. Representative images (10x) and bar graphs indicating the relative area and width of the scratch at $0 \mathrm{~h}, 12 \mathrm{~h}$, and $24 \mathrm{~h}$ were shown. (C-F) The SK-N-SH cell's invasion as well migration was analyzed using transwell assay after the treatment with DHA. The cell numbers in five stochastic microscopic fields (100x) was calculated per group. $(\mathbf{G})$ and $(\mathrm{H})$ Western blotting of EMT 
proteins levels in cells of SK-N-SH was performed after the different indicated treatment. All data are represented as mean $\pm S D$; NC: negative control, mi: mimic, in: inhibitor.
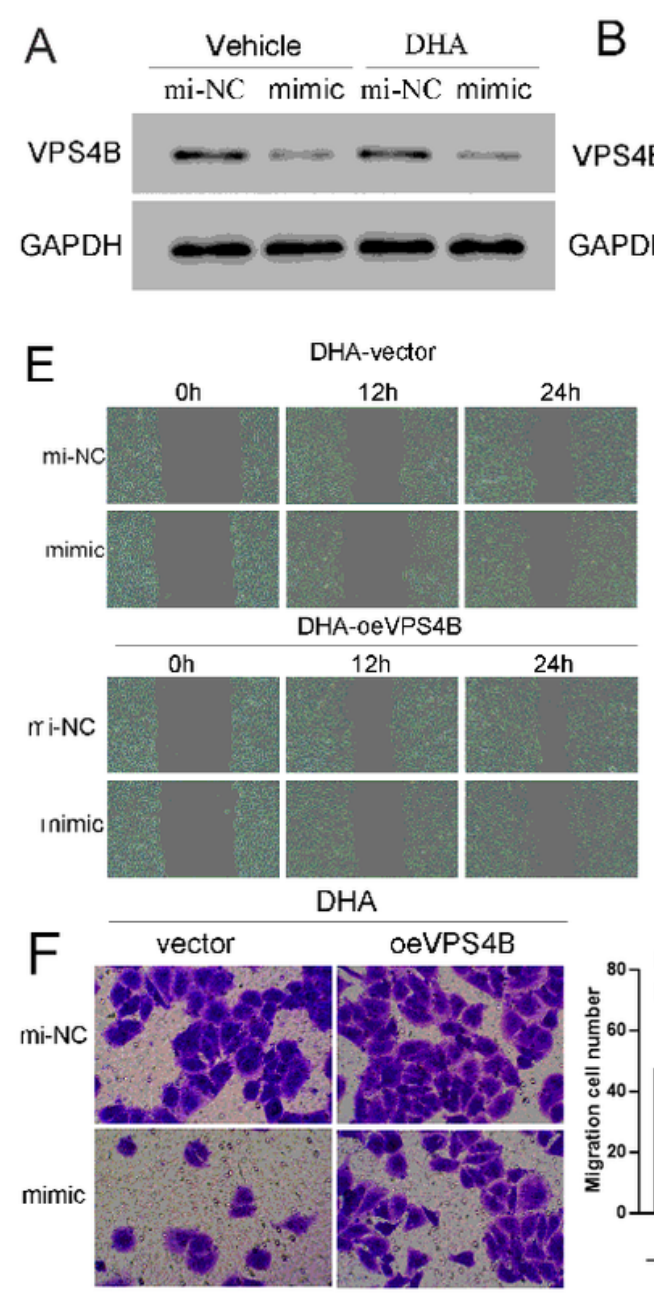

B Vehicle DHA in-NC inhibitor in-NC inhibitor

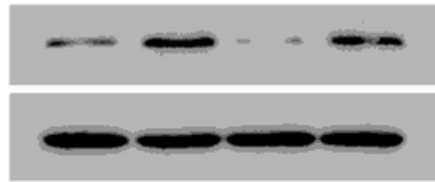

C
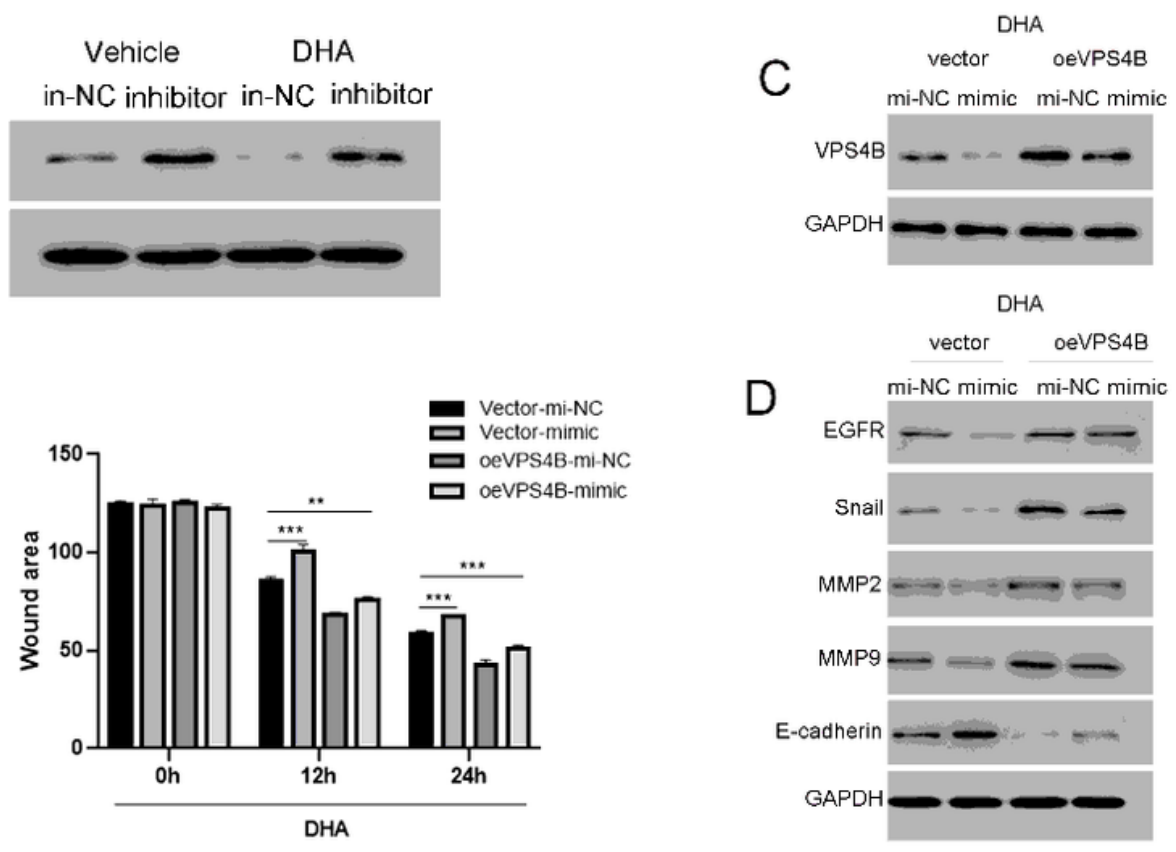

D
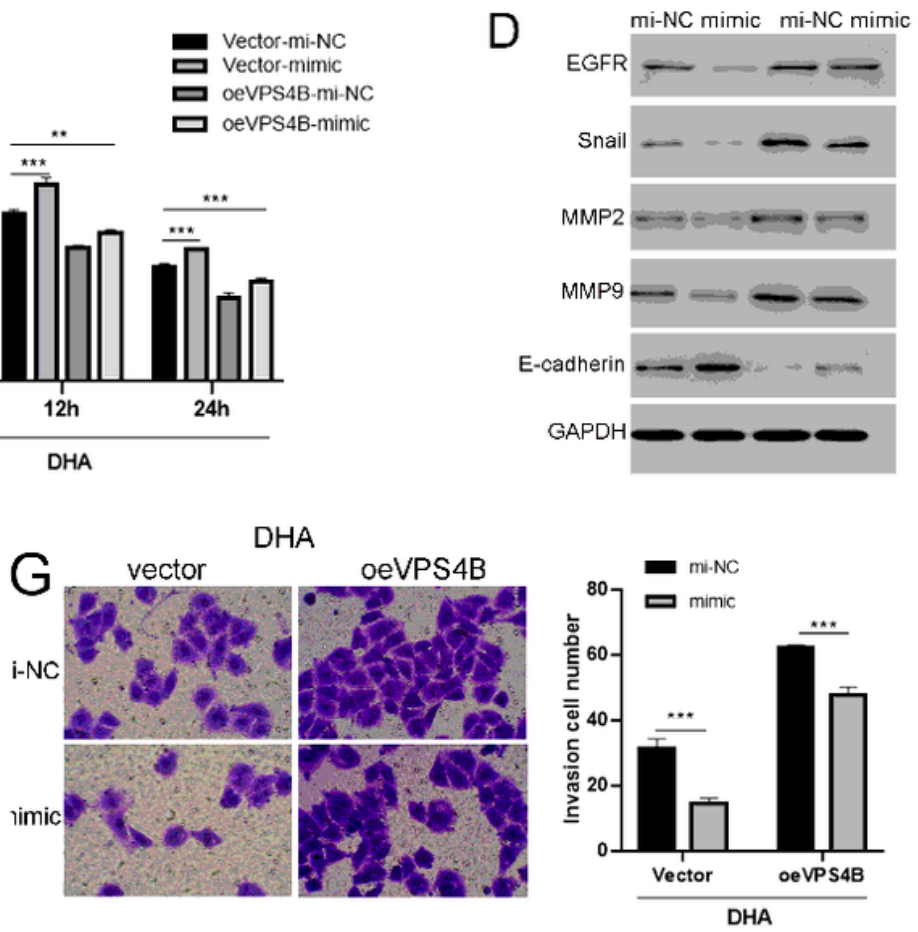

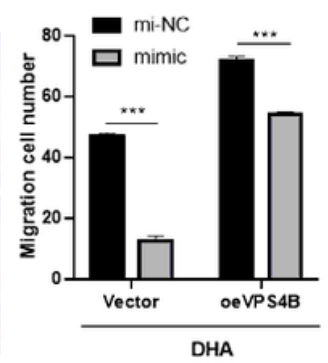

\section{Figure 6}

VPS4B controls the cell migration and its invasion and functions in the synergistic therapy roles of miR32-5b mimic and DHA. The level of protein expression of VPS4B changes was measured in cells of SK-NSH with miR-32-5p mimic (A) and in cells of IMR-32 with miR-32-5p inhibitor (B). (C) Western blotting of VPS4B in SK-N-SH cells expressing VPS4B or empty vector constructs with miR-32-5p mimic or NC. The cell was subjected to transfection with miR-32-5p and transfected with VPS4B overexpression plasmid (oeVPS4B). (D) Western blotting analysis of EGFR, Snail, MMP2, MMP9, and E-cadherin in SK-N-SH cells expressing VPS4B or empty vector constructs with miR-32-5p mimic or NC. The cell was subjected to transfection miR-32-5p and transfected with oeVPS4B. GAPDH was taken as a control. (E) Wound healing analysis was carried out in cells with VPS4B-overexpressing or cells with the control vector. Transwell migration (F) as well as invasion (G) assay was carried out in cells with VPS4B-overexpressing or cells with control vector. Five stochastic microscope ranges were observed to count the cell numbers per group. All data are represented as mean \pm SD. NC: negative control, mi: mimic, in: inhibitor, oe VPS4B: overexpression of VPS4B. 
A

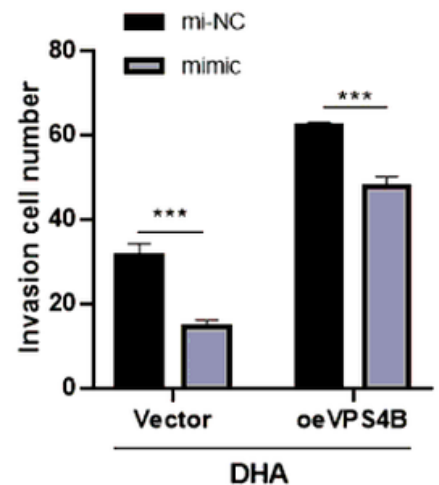

C

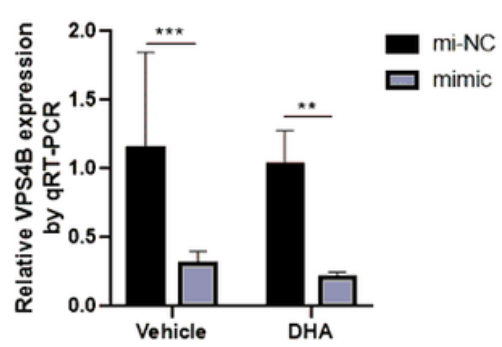

B

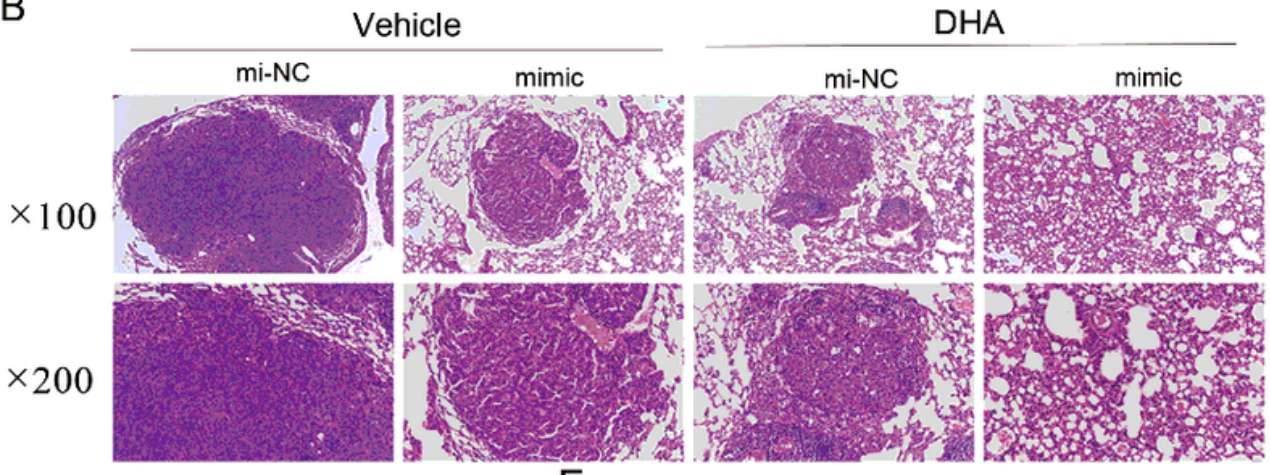

$\mathrm{F}$

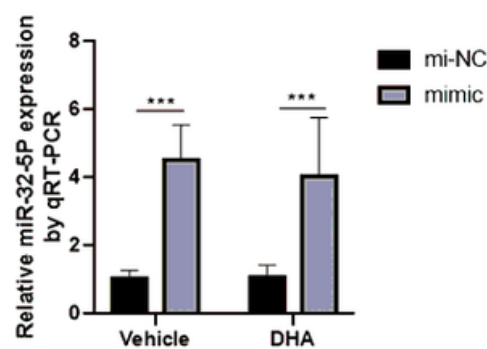

$\mathrm{DHA}$
$E$

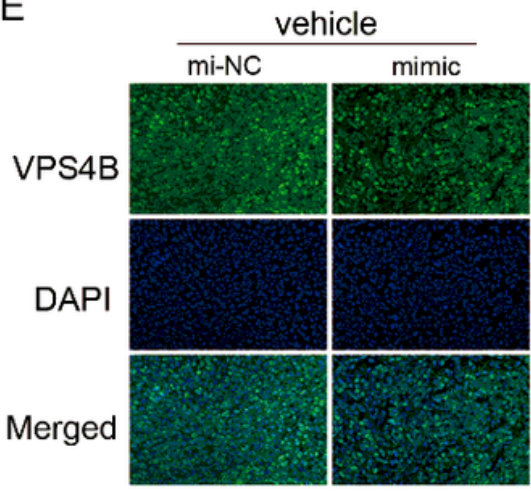

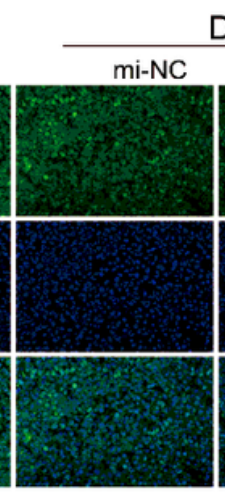

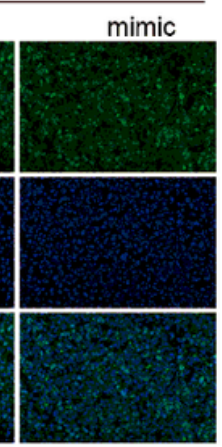

Figure 7

The function of miR-32-5p on metastasis of NB tumor cells was confirmed by in vivo studies. (A) Quantification of lung metastases of the mi-NC and mimic with or without DHA treatment. (B) The metastasis in mice was determined by HE staining. The representative images (100x, upper panel and 200x, lower panel). (C) and (D) The miR-32-5p and VPS4B mRNA expression in tissues of cancer. (E) Representative photos of immunofluorescent staining for VPS4B in the four mouse groups $(4 \times)$. (F) Western blotting of VPS4B expression in metastatic nodes. (G) Western blotting of EGFR, Snail, MMP2, MMP9, and E-cadherin expression in metastasis nodes. GAPDH was taken as an inner control. Data are represented as mean \pm SD. mi-NC: mimic-negative control.

\section{Supplementary Files}

This is a list of supplementary files associated with this preprint. Click to download.

- Supplementaryfigures.pdf 